\title{
Search for comet-like activity in asteroid 7968 Elst-Pizarro and limitation of its rotational pole orientation
}

\author{
I. Toth
}

Konkoly Observatory, Budapest, 1525 PO Box 67, Hungary

e-mail: tothi@konkoly.hu

Received 1 June 2005 / Accepted 1 September 2005

\section{ABSTRACT}

We present the results of a new investigation of the asteroid-comet transition object or activated asteroid 7968 Elst-Pizarro (133P/Elst-Pizarro). We performed Cousins $R$-band CCD imaging observations on UT 16 January, 28 February and 1 March 2005 in order to search for comet-like activity of this activated asteroid. These observations do not show any comet-like activity shuch as coma, tail(s) or a dust trail. The object was at large heliocentric distances between 3.623 and 3.647 AU a few months after its aphelion. Taking into account the seasonal modulation model to explain the activity, the distribution of the active/inactive phases along its orbit, and the available lightcurve observations of Elst-Pizarro, we propose four working models for the possible orientation of its rotational pole. These four best fit working models for the pole orientation of Elst-Pizarro are in a good agreement with the observed data. The true direction of the rotational pole is still unknown.

Key words. minor planets, asteroids - comets: individual: 133P/Elst-Pizarro - comets: general - techniques: image processing

\section{Introduction}

There are asteroids which display temporary comet-like activity. These are the asteroid-comet transition objects which can be found among the near-Earth objects, centaurs, transneptunian objects, and the damocloids - asteroids in comet-like orbits which are associated with the Oort cloud (Jewitt 2005). There are two well known examples of this behavior: 4015 Wilson-Harrington and 7968 Elst-Pizarro showing a temporary dust tail and trail (Elst et al. 1996; Fernández et al. 1997; Hsieh et al. 2003, 2004, see also the reviews by Jewitt 2004; and Weissman et al. 2002). These two objects are also cataloged as comets: 107P/Wilson-Harrington and 133P/Elst-Pizarro (hereafter E-P in short) but they have a typically asteroid orbit with value of the Tisserand parameter with respect to Jupiter of 3.084 for $107 \mathrm{P}$ and 3.184 for E-P.

In this paper we study Elst-Pizarro. This object displays a narrow linear dust feature trailing the object which led to its serendipitous recovery in 1996 and it was designed as comet P/1996 N2 (Elst et al. 1996). Shortly after it was determined that it had already been discovered in 1979 as a mainbelt asteroid 1979 OW7 (Marsden 1996; Marsden \& McNaught 1996; McNaught et al. 1996) but at that time it was in its asteroid form and it did not show any comet-like activity; the same occurred in 1985 (McNaught et al. 1996). But there was no any gas activity (gas coma and/or spectral features) reported in 1996, and the only dominating comet-like feature was the observed straight line dust tail. Efforts were carried out to explain E-P's enigmatic comet-like behavior: Boehnhardt et al. (1998), Lien (1998), Toth (2000) assumed that recent impact event disturbed the surface and generated ejection of surface dust material. This process can occur assuming either the existence of a subsurface volatile component which drives the dust, i.e., volatile-driven mass-loss activity (Boehnhardt et al. 1998; Lien 1998) or the dust production might have resulted from impacts onto E-P that generated a temporary dust tail or trail; this case does not need the presence of an interior volatile component (Toth 2000).

$B V R I$ observations of E-P obtained by Delahodde et al. (2004) at the ESO with the 3.6-m NTT in 2000 are consistent with a dormant (inactive) phase of this object, as no coma or tail (trail) was detected to $25 \mathrm{mag} / \mathrm{sq}$. arcsec. The observations of E-P made by Hsieh et al. (2004) with the UH 2.2-m telescope in 2002 witnessed the return of its new dust trail (without a trace of gas activity), but in 2003 they did not find any comet-like activity despite using the Keck I 10-m telescope. The one-time impact event hypotheses proposed to explain the 1996 apparition of a comet-like tail was since ruled out by the discovery of recurrent activity in 2002. This result reopened the debate about the nature of E-P's dust-emission activity and the physical characteristics and origin of E-P itself. The existence and behavior of E-P pose serious problems for our understanding of comets and asteroids and how they relate to one another. If E-P is a comet then its orbit is particularly unusual as it has a typical low-eccentricity, low-inclination, main-belt asteroid orbit $\left(a=3.158 \mathrm{AU}, e=0.165, i=1.38^{\circ}\right)$. This orbit places it in 
Table 1. Journal of observations of 7968 Elst-Pizarro.

\begin{tabular}{|c|c|c|c|c|c|c|c|c|c|c|}
\hline $\begin{array}{c}\text { Image } \\
\text { No. }\end{array}$ & $\begin{array}{l}\text { UT Date } \\
2005 \ldots\end{array}$ & Filter & $\begin{array}{c}\text { Exposure } \\
\text { (s) }\end{array}$ & $\begin{array}{l}\text { Pixel } \\
(\mathrm{km})\end{array}$ & Moon & Weather & $\begin{array}{c}\text { Seeing } \\
(\operatorname{arcsec})\end{array}$ & Air mass & $\begin{array}{c}\text { Apparent } \\
R \text { (mag) }\end{array}$ & $\begin{array}{l}\text { Tail/trail } \\
\text { detection }\end{array}$ \\
\hline \multicolumn{11}{|c|}{ Night of 15/16 January: $r_{\mathrm{h}}=3.647 \mathrm{AU}, \Delta=2.700 \mathrm{AU}, \alpha=4.85^{\circ}$} \\
\hline$\# 1$ & January 16.105 & $R$ & 500 & 593 & $\mathrm{~N}+5$ & haze & 2.0 & 1.19 & 20.47 & No \\
\hline$\# 2$ & January 16.115 & $R$ & 500 & 593 & $\mathrm{~N}+5$ & haze & 2.0 & 1.20 & 20.47 & No \\
\hline \multicolumn{11}{|c|}{ Night of 28 February/1 March: $r_{\mathrm{h}}=3.624 \mathrm{AU}, \Delta=2.761 \mathrm{AU}, \alpha=8.82^{\circ}$} \\
\hline \#3 & February 28.934 & $4 \quad R$ & 1000 & 607 & $\mathrm{~N}+20$ & cirrus & 2.0 & 1.16 & 20.68 & No \\
\hline \#4 & February 28.948 & $R$ & 1200 & 607 & $\mathrm{~N}+20$ & cirrus & 2.0 & 1.15 & 20.68 & No \\
\hline \multicolumn{11}{|c|}{ Night of $1 / 2$ March: $r_{\mathrm{h}}=3.623 \mathrm{AU}, \Delta=2.769 \mathrm{AU}, \alpha=9.08^{\circ}$} \\
\hline \#5 & March 1.986 & $R$ & 1200 & 609 & $\mathrm{~N}+21$ & clear & 2.0 & 1.19 & 20.70 & No \\
\hline \#6 & March 1.986 & $R$ & 1200 & 609 & $\mathrm{~N}+21$ & clear & 2.0 & 1.22 & 20.70 & No \\
\hline
\end{tabular}

Note:

$r_{\mathrm{h}}, \Delta$ : helio- and geocentric distances, $\alpha$ : solar phase angle (Sun-object-Earth angle).

Moon: Lunar phase expressed in offset from the new Moon ("N") in days.

* Apparent $R$ magnitude was computed from the effective radius, geometric albedo, and linear phase coefficient determined by Hsieh et al. (2004).

the Themis collisional asteroid family. It is highly improbable that an extinct comet would evolve to such an orbit (Weissman et al. 2002) unless the nongravitational forces or Yarkovsky effect act upon it effectively (Hsieh et al. 2004). If E-P is an asteroid then its physical nature is unusual because this object at times shows comet-like activity by developing a narrow dust tail or trail whilst there is no detectable gas activity.

However, Lowry \& Fitzsimmons (2005) published their 2002 observations of E-P, which were made with the 4.2-m William Herschel Telescope (WHT) on 13 July 2002 and they reported very little dust activity with a small dust trail (or tail). They proposed that the evolution of the dust trail started just prior to their observations in July 2002. They will give a more detailed analysis of their E-P observations in the future.

Hitherto the most comprehensive and observational evidence based detailed exploration of E-P has been carried out by Hsieh et al. (2004). They suggested various conceptions to explain the observed recurrent comet-like behavior of E-P. According to Hsieh et al. (2004) the most preferable idea is that if the 1996 and 2002 emission events are consequences of seasonal insolation variation, the area of exposed surface volatiles must be located near one of the rotational poles of the nucleus and the object's obliquity angle, i.e., the tilt between its equatorial and orbital planes, must be nonzero. This volatile region would then only receive enough solar radiation to become active when the near pole tilts toward the Sun, during hemisphere's "summer". This object can be termed as "activated asteroid" which remains consistent with the possibility of seasonal modulation of E-P's activity (R.P. Binzel, cf. Hsieh et al. 2004). The question is raised as to where E-P exhibited comet-like activity and where it was inactive along its orbit, i.e., how long the periods of activity and dormancy were. For this purpose we continued the search for comet-like activity and in this paper we present our results obtained by CCD imaging observations of this object conducted in January and February of
2005 when E-P was close to its opposition (Sect. 2). In addition, mapping of the location of the active and inactive phases of E-P along its orbit (Sect. 3) and taking into account the lightcurve observations now available in the literature we attempt to estimate the limitations of the possible rotational pole orientation of this object (Sect. 4).

\section{Observations at Konkoly Observatory in 2005}

CCD imaging observations were performed in the $R$ band of Cousins photometric system on the following nights in 2005: UT 15/16 January, 28 February/1 March, and 1/2 March (Table 1, Figs. 1a-c). The CCD camera was attached to the Cassegrain focus of the 1-m Ritchey-Chrétien-Coudé (RCC) telescope at Piszkéstetö station (960 m altitude) of the Konkoly Observatory. The CCD camera was Roper Scientific VersArray 1300B of Princeton Instruments, which has a backilluminated Marconi CCD36-40 scientific grade image sensor and it is coated for extended UV response. It is a high-performance, back-illuminated full-frame digital camera system, which offers the highest sensitivity from the UV to the near infrared. The chip format is $20 \times 20$ micron sized imaging pixels in $1340 \times 1300$ imaging array with $100 \%$ fill factor and optically centered $26.8 \times 26.0 \mathrm{~mm}$ imaging area. The focal length of the telescope is $13.6 \mathrm{~m}$ and the corresponding scale is $0.303 \mathrm{arcsec} / \mathrm{pixel}$. This system provides a very large imaging area with very high spatial resolution: in this system configuration the total field-of-view (FOV) is $6.77^{\prime} \times 6.57^{\prime}$. Typical read noise of the CCD is 2 electrons and the system typical read noise is 2.8 electrons at $50 \mathrm{kHz}$. The typical value of the singlepixel full well is $300 \mathrm{ke}$, the dark current at $-40 \mathrm{C}(233 \mathrm{~K})$ is $0.1 \mathrm{e} / \mathrm{pixel} / \mathrm{s}$, the nonlinearity is less than $2 \%$. The operational temperature of CCD was $-41 \mathrm{C}(232 \mathrm{~K})$ on each night of the observations. The telescope operated at sidereal rate and in the CCD frames the object was slightly trailed due to the 


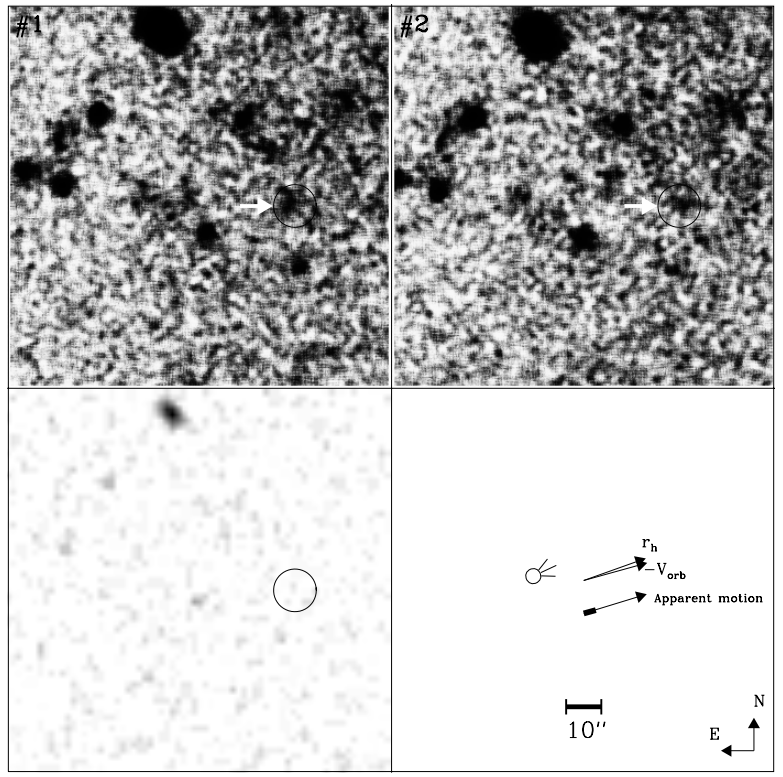

a) Observational night of 15/16 January 2005 .

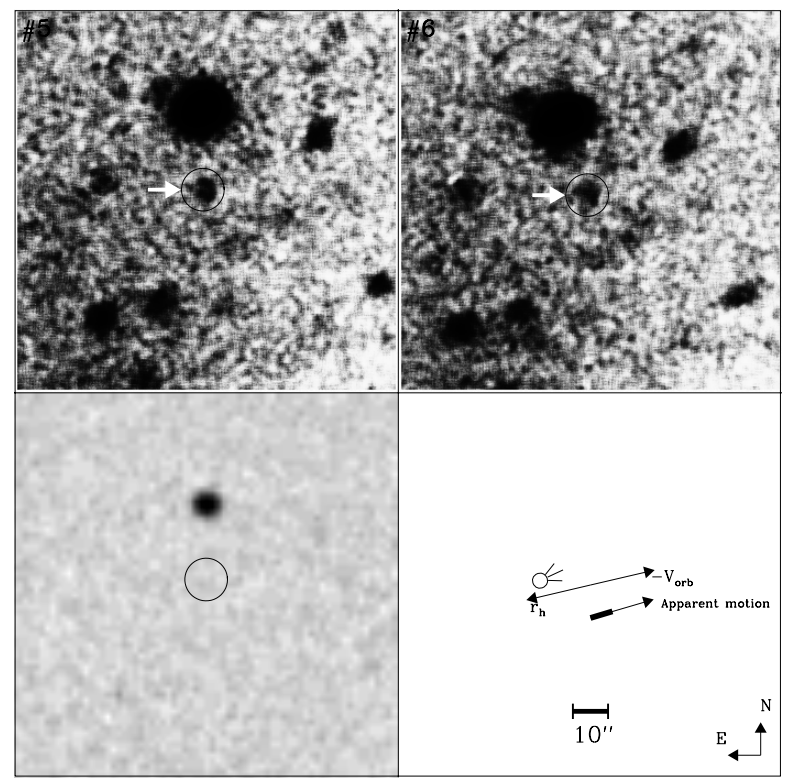

c) Observational night of 1/2 March 2005 .

long exposures of 500, 600, 1000 and $1200 \mathrm{~s}$ (Table 1). The observations were made between zenith angles of $30^{\circ}$ and $33^{\circ}$ at an airmass of 1.14-1.22 when the object was close to its upper culmination. Standard reduction procedures were applied for the CCD images including bias correction and flat-fielding. The dark current is negligible with this CCD camera for exposure times of less than about five minutes but the dark frames were applied during the reduction due to the applied long exposures. Figures 1a-c display the $R$ observed images with the corresponding POSS1 Red sky survey maps around the location of the object.

We observed this object when it was at large heliocentric distances between $r_{\mathrm{h}}=3.647 \mathrm{AU}$ and 3.623 $\mathrm{AU}$ on the inbound part of its orbit, and between geocentric distances $\Delta=2.700 \mathrm{AU}$ and $2.769 \mathrm{AU}$, and at a solar phase angles of $4.85^{\circ}-9.08^{\circ} .7968$ E-P was in aphelion on

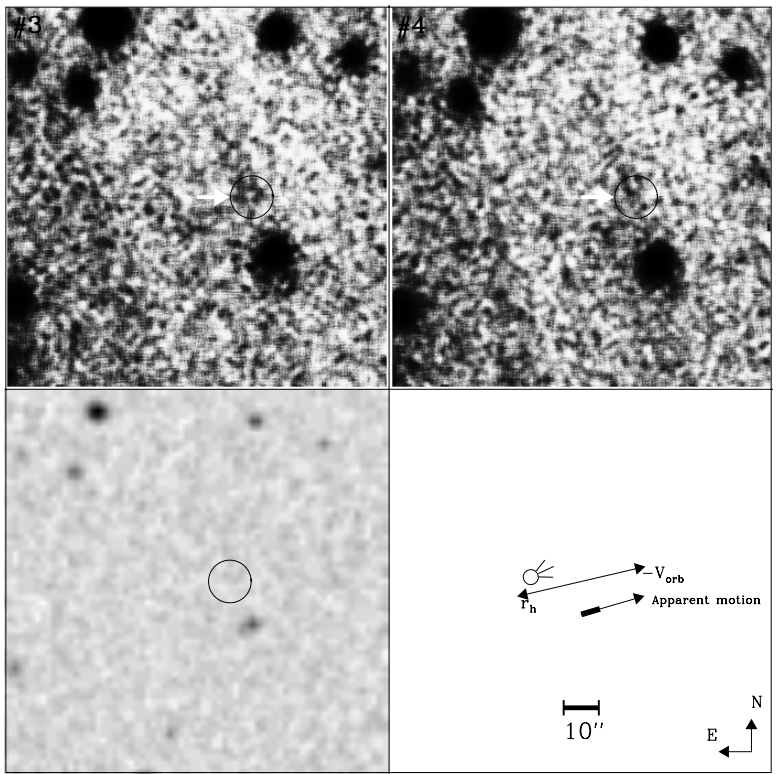

b) Night of 28 February/1 March 2005 .

Fig. 1. Subframes extracted from the CCD images of the 7968 Elst-Pizarro taken with $R$ filter on the nights of UT 15/16 January, 28 February/1 March, and 1/2 March 2005 are displayed (top panels: left and right, respectively, see Table 1). An arrow points the object in both subframes (the location of the object is surounded by a circle). For a comparison, the POSS1 red sky map image of the same star field is displayed (bottom left panel) on the same scale as of the observed images. The celestial equatorial North and East directions are marked by arrows (bottom left panel). The arrows indicate the anti-solar direction (prolonged radius vector $\boldsymbol{r}_{\mathrm{h}}$ ), the opposite direction of the heliocentric orbital velocity vector $\left(-\boldsymbol{V}_{\text {orb }}\right)$, and the direction of the apparent motion of the object projected onto the sky plane. The length of the trail of apparent motion during the expositions $(500,1000$, or $1200 \mathrm{~s}$ each) is drawn by a solid line interval along the vector of the apparent motion direction. Comet symbol oriented in the expected trail (or trail) direction $\left(-\boldsymbol{V}_{\text {orb }}\right)$ in case of emission of dust grains with small values of radiation pressure parameter $\beta$. Angular scale bar of 10 arcsec is also marked.

UT 11 September 2004 at $Q=3.676$ AU heliocentric distance. The object was in opposition on UT 31 January 2005. Our first observation was taken 127 days after aphelion and 15 days before opposition. Table 1 lists the projected linear native pixel size which was between $593 \mathrm{~km}$ and $609 \mathrm{~km}$ at the actual geocentric distance of the object. Other angular data of the aspect geometry are shown in Table 2 . The radius vector inclined to the plane of FOV at the comet with angle between $81^{\circ}$ and $86^{\circ}$ (close to the direction of line-of-sight), and the negative heliocentric orbital velocity vector inclined to the plane of FOV between $-1^{\circ}$ (inward to the observer) and $14^{\circ}$ (outward from the observer). The angle between the line-of-sight and the orbital plane of the object is also listed as the orbit-plane angle.

Inspection of the images obtained show that E-P was very faint and did not exhibit any comet-like activity (Fig. 1). Despite the faintness of E-P, which has $\sim 20.5 \mathrm{mag}$ in $R$ at the 
Table 2. Journal of detected or non-detected comet-like activity in asteroid-comet transition object 7968 Elst-Pizarro.

\begin{tabular}{|c|c|c|c|c|c|c|c|c|c|c|c|c|c|c|}
\hline UT Date & Telescope & Ref. & $\begin{array}{c}\text { Flag of } \\
\text { detection }\end{array}$ & $\begin{array}{r}f \\
\left({ }^{\circ}\right) \\
\end{array}$ & $\begin{array}{r}L \\
\left({ }^{\circ}\right) \\
\end{array}$ & $\begin{array}{r}B \\
\left({ }^{\circ}\right) \\
\end{array}$ & $\begin{array}{c}r_{\mathrm{h}} \\
(\mathrm{AU}) \\
\end{array}$ & $\begin{array}{c}\Delta \\
(\mathrm{AU})\end{array}$ & $\begin{array}{r}\alpha \\
\left({ }^{\circ}\right) \\
\end{array}$ & $\begin{array}{r}\alpha_{\mathrm{pl}} \\
\left(^{\circ}\right) \\
\end{array}$ & $\begin{array}{r}\mathrm{PA}\left(\boldsymbol{r}_{\mathrm{h}}\right) \\
\left({ }^{\circ}\right)\end{array}$ & $\begin{array}{c}\left(\boldsymbol{r}_{\mathrm{h}}, \mathrm{FOV}\right) \\
\left(^{\circ}\right)\end{array}$ & $\begin{array}{c}\mathrm{PA}(-\boldsymbol{V}) \\
\left(^{\circ}\right)\end{array}$ & $\begin{array}{c}(-\boldsymbol{V}, \mathrm{FOV}) \\
\left(^{\circ}\right)\end{array}$ \\
\hline 19790724 & UK Schmidt $1.2 \mathrm{~m}$ & $\mathrm{McN}+96$ & NO & 8.0 & 301.0 & 0.9 & 2.64 & 1.62 & 1.5 & -0.6 & 101 & 90 & 260 & -1 \\
\hline 19850915 & UK Schmidt $1.2 \mathrm{~m}$ & $\mathrm{McN}+96$ & NO & 54.8 & 347.7 & -0.2 & 2.80 & 1.80 & 3.1 & 0.2 & 66 & 88 & 249 & -4 \\
\hline 19960714 & ESO $1.0 \mathrm{~m}$ & E+96 & YES & 22.2 & 315.2 & 0.6 & 2.66 & 1.78 & 13.1 & -0.6 & 247 & 77 & 253 & -16 \\
\hline 19960809 & ESO $1.0 \mathrm{~m}$ & $\mathrm{E}+96$ & YES & 28.5 & 321.5 & 0.4 & 2.68 & 1.67 & 2.9 & -0.3 & 253 & 88 & 254 & -6 \\
\hline 19960821 & Klet $0.57 \mathrm{~m}$ & TT96 & YES & 31.4 & 324.4 & 0.4 & 2.69 & 1.68 & 2.4 & -0.2 & 102 & 88 & 255 & -1 \\
\hline 19960821 & Cloudcroft $0.6 \mathrm{~m}$ & O96 & YES & 31.4 & 324.4 & 0.4 & 2.69 & 1.68 & 2.4 & -0.2 & 102 & 88 & 255 & -1 \\
\hline 19960822 & Ondrejov $0.65 \mathrm{~m}$ & P96 & YES & 31.6 & 324.6 & 0.4 & 2.69 & 1.69 & 2.8 & -0.2 & 101 & 88 & 255 & -1 \\
\hline 19960916 & $\mathrm{ESO} 2.2 \mathrm{~m}$ & $\mathrm{~B}+96$ & YES & 37.5 & 330.5 & 0.2 & 2.71 & 1.83 & 12.4 & 0.2 & 92 & 78 & 256 & 8 \\
\hline 19960918 & Apache Point $3.5 \mathrm{~m}$ & H96 & YES & 38.0 & 331.0 & 0.2 & 2.72 & 1.85 & 13.0 & 0.2 & 92 & 78 & 256 & 8 \\
\hline 19961004 & ESO Danish $1.54 \mathrm{~m}$ & $\mathrm{~B}+96$ & YES & 41.7 & 334.7 & 0.1 & 2.73 & 2.02 & 17.1 & 0.4 & 89 & 73 & 256 & 12 \\
\hline 19971001 & ESO $2.2 \mathrm{~m}$ & B97 & NO & 112.2 & 45.2 & -1.3 & 3.27 & 2.55 & 13.8 & 0.3 & 252 & 77 & 254 & -23 \\
\hline 19971003 & Cloudcroft $0.6 \mathrm{~m}$ & O97 & NO & 112.5 & 45.5 & -1.3 & 3.28 & 2.53 & 13.3 & 0.3 & 252 & 77 & 254 & -22 \\
\hline 19971004 & Cloudcroft $0.6 \mathrm{~m}$ & O97 & NO & 112.7 & 45.6 & -1.3 & 3.28 & 2.52 & 13.1 & 0.3 & 252 & 77 & 254 & -22 \\
\hline 20000401 & ESO $3.6 \mathrm{~m} \mathrm{NTT}$ & $\mathrm{D}+04$ & NO & 237.4 & 170.4 & 0.2 & 3.37 & 2.46 & 8.4 & -0.3 & 102 & 82 & 292 & 18 \\
\hline 20000505 & ESO $3.6 \mathrm{~m}$ NTT & $\mathrm{D}+04$ & NO & 242.7 & 175.6 & 0.4 & 3.32 & 2.77 & 16.0 & -0.5 & 106 & 74 & 291 & 26 \\
\hline 20020713 & WHT $4.2 \mathrm{~m}$ & LF05 & YES & 55.4 & 348.4 & -0.2 & 2.81 & 2.42 & 20.8 & -0.4 & 247 & 70 & 249 & -27 \\
\hline 20020819 & $\mathrm{UH} 2.2 \mathrm{~m}$ & HJF04 & YES & 63.4 & 356.3 & -0.4 & 2.86 & 2.05 & 14.5 & -0.2 & 248 & 76 & 249 & -22 \\
\hline 20020907 & $\mathrm{UH} 2.2 \mathrm{~m}$ & HJF04 & YES & 67.3 & 0.3 & -0.5 & 2.89 & 1.94 & 8.3 & 0.0 & 249 & 82 & 249 & -16 \\
\hline 20020908 & $\mathrm{UH} 2.2 \mathrm{~m}$ & HJF04 & YES & 67.6 & 0.5 & -0.5 & 2.89 & 1.93 & 7.9 & 0.1 & 249 & 83 & 248 & -16 \\
\hline 20020909 & $\mathrm{UH} 2.2 \mathrm{~m}$ & HJF04 & YES & 67.8 & 0.7 & -0.5 & 2.89 & 1.93 & 7.6 & 0.1 & 249 & 83 & 248 & -15 \\
\hline 20021105 & $\mathrm{UH} 2.2 \mathrm{~m}$ & HJF04 & YES & 79.2 & 12.2 & -0.7 & 2.98 & 2.18 & 13.2 & 0.6 & 66 & 77 & 248 & 5 \\
\hline 20021106 & $\mathrm{UH} 2.2 \mathrm{~m}$ & HJF04 & YES & 79.4 & 12.4 & -0.7 & 2.98 & 2.19 & 13.5 & 0.6 & 66 & 77 & 248 & 5 \\
\hline 20021107 & $\mathrm{UH} 2.2 \mathrm{~m}$ & HJF04 & YES & 79.6 & 12.6 & -0.7 & 2.98 & 2.20 & 13.7 & 0.6 & 66 & 77 & 248 & 5 \\
\hline 20021227 & $\mathrm{UH} 2.2 \mathrm{~m}$ & HJF04 & YES & 89.1 & 22.0 & -0.9 & 3.06 & 2.93 & 18.7 & 0.4 & 67 & 72 & 248 & 10 \\
\hline 20021228 & $\mathrm{UH} 2.2 \mathrm{~m}$ & HJF04 & YES & 89.2 & 22.2 & -0.9 & 3.06 & 2.94 & 18.7 & 0.4 & 67 & 72 & 248 & 10 \\
\hline 20030922 & Keck I 10 m & HJF04 & NO & 132.7 & 65.7 & -1.4 & 3.46 & 3.20 & 16.8 & 0.1 & 266 & 74 & 259 & -24 \\
\hline 20050116 & Piszkéstetö $1 \mathrm{~m}$ & This work & NO & 196.3 & 129.3 & -0.7 & 3.65 & 2.70 & 4.8 & 0.4 & 292 & 86 & 283 & -1 \\
\hline 20050301 & Piszkéstetö $1 \mathrm{~m}$ & This work & NO & 202.0 & 135.0 & -0.6 & 3.62 & 2.76 & 8.9 & -0.0 & 80 & 82 & 281 & 14 \\
\hline 20050302 & Piszkéstetö $1 \mathrm{~m}$ & This work & NO & 202.2 & 135.2 & -0.6 & 3.62 & 2.77 & 9.1 & -0.0 & 80 & 81 & 281 & 14 \\
\hline
\end{tabular}

References:

B+96: Boehnhardt et al. (1996); B97: Boehnhardt (1997); D+04: Delahodde et al. (2004); E+96: Elst et al. (1996);

H96: Hammergren (1996); HJF04: Hsieh et al. (2004); LF05: Lowry \& Fitzsimmons (2005); McN+96: McNaught et al. (1996);

O96: Offutt (1996); O97: Offutt (1997); P96: Pravec (1996); TT96: Tichá \& Tichý (1996).

Note:

$f$ : true anomaly; $L, B$ : heliocentric longitude and latitude; $r_{\mathrm{h}}, \Delta$ : helio- and geocentric distances; $\alpha$ : solar phase angle (Sun-object-Earth angle); $\alpha_{\mathrm{pl}}$ : orbit-plane angle (between observer and object orbit plane as seen from the object);

$\mathrm{PA}\left(r_{\mathrm{h}}\right)$ : position angle of the radius vector; $\left(r_{\mathrm{h}}, \mathrm{FOV}\right)$ : angle between field-of-view and the radius vector;

$\mathrm{PA}(-V)$ : position angle of the opposite heliocentric orbital velocity vector;

$(-V$, FOV $)$ : angle between field-of-view and the opposite heliocentric orbital velocity vector.

*1-m Ritchey-Chrétien-Coudé (RCC) telescope of Konkoly Observatory at Piszkéstetö.

time of observations (Table 1), we expected to see the at least the beginning of the trail, i.e., elongated shape or diffuse appearance of the object. If there were any coma or trail these had to be observed at least at close to the peak pixel (optocenter) of E-P because the brightest part of either the coma or trail have comparable brightness to the peak pixel as can be seen in Figs. 4-7 of Hsieh et al. (2004) if we assume a similar brightness distribution during our observations as it was in 2002. Comparison of the linear $X$ and $Y$ profiles of the images of the object are displayed in Fig. 2. The background limit was $\sim 22 \mathrm{mag} / \square^{\prime \prime}$ in the observed images. In order to increase the signal-to-noise ratio we also studied the coadded $R$ filtered 


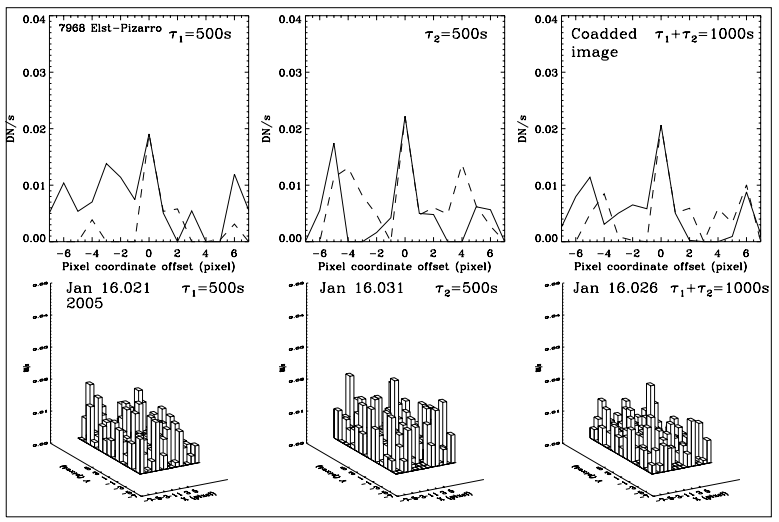

a) Observational night of 15/16 January 2005 .

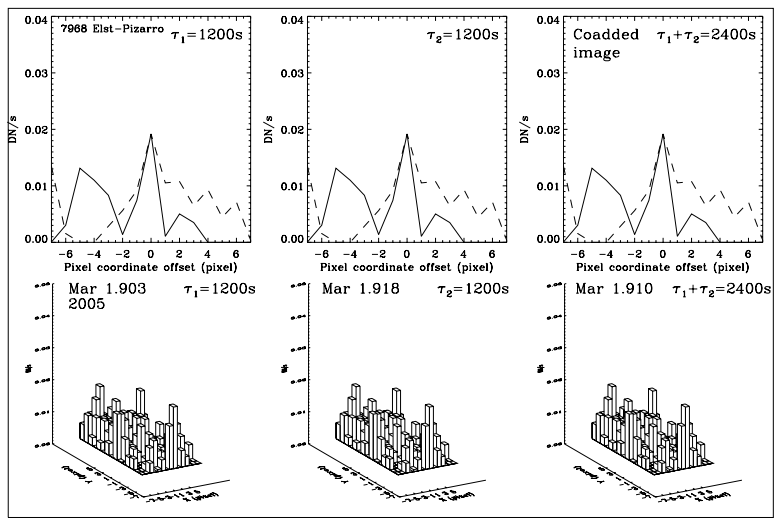

c) Observational night of 1/2 March 2005 .

images made on the same night. The profile slices cut through the peak pixel (optocenter) of the object in horizontal $(X)$ and vertical $(Y)$ directions did not show any trace of coma or trail (tail) for either the individual images or coadded image composed from the consecutive images made on the same night. Thus, our recent observations of E-P do not show any cometlike activity in the inbound part of its orbit and support the model of the seasonally activated asteroid.

\section{Distribution of the active and dormant phases in the orbit of Elst-Pizarro}

The journal of detected or non-detected comet-like activity in Elst-Pizarro is listed in Table 2. True anomaly and other parameters of the aspect geometry are also calculated for the time of the observations using the ASTORB data base of osculating elements of asteroids which is maintained by E.L.G. Bowell of the Lowell Observatory. Since we are interested in the positions of the active and dormant phases along the orbit of E-P we plotted the reported observations. In this manner we can compare the active and dormant phases of E-P as these are displayed in Fig. 3. The background objects are the Themis zone asteroids and the orbit of 7968 Elst-Pizarro resides clearly in this region of the outer main belt. The 1979 discovery observation was just after the perihelion of E-P and the nearest observed active phase was observed in 1996. Locations of the active periods aligne along the post-perihelion arc between true anomalies $22^{\circ}$ and $89^{\circ}$ bracketed by the 1996 and

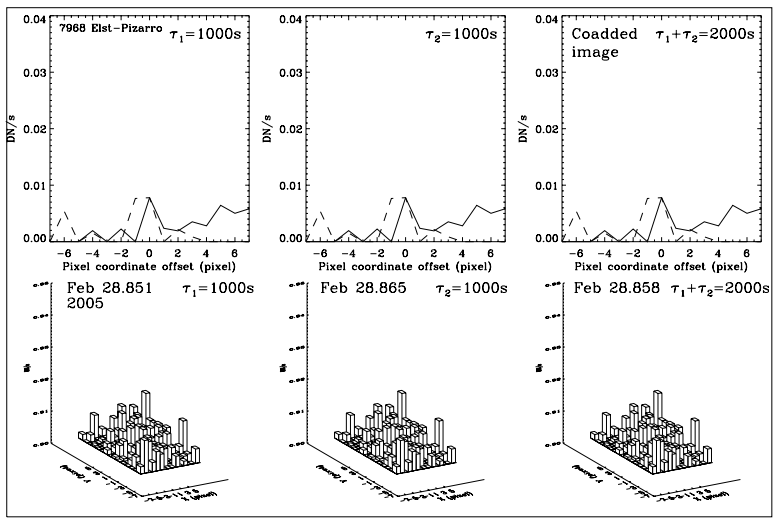

b) Observational night of 28 February/1 March 2005 .

Fig. 2. Comparison of the linear profiles of the observed images of 7968 Elst-Pizarro made on the nights of UT 15/16 January, 28 February/1 March, and 1/2 March 2005. Profile slices cut through the peak pixel (optocenter) of the object along horizontal $X$ (solid line) and vertical $Y$ (dashed line) directions (top left and mid panels), as well as of the coadded image (top right panel). The horizontal $(X)$ and vertical $(Y)$ profiles are nearly symmetric, indicating that the object was close to the center of the pixel. The 2D stacked histogram-style plot represents the corresponding subframes of the observed images centered on the peak pixels (optocenters), which are displayed in the three bottom panels. Background has been subtracted. Units of the $X, Y$ axes are in pixel coordinates and the pixel values are in $\mathrm{DN} / \mathrm{s}$.

2002 observations (Hsieh et al. 2004; Lowry \& Fitzsimmons 2005). Lowry \& Fitzsimmons (2005) reported activity of E-P in 2002, but at almost the same position of the orbit McNaught et al. (1996) did not observe any comet-like activity in 1985 when E-P was asteroidal and trailed. We suspect that there is not enough angular resolution and sufficiently high signalto-noise ratio in those Schmidt photographic plates of the 1985 observations to detect comet-like activity in the trailed images of E-P. However, in 2002 Lowry \& Fitzsimmons (2005) observed the activity using advanced techniques (4.2-m WHT and CCD). Comet-like activity of E-P was detected in this postperihelion orbit arc exclusively. Outside this post-perihelion orbit arc the comet-like activity has not been detected (McNaught et al. 1996; Offutt 1996, 1997; Delahodde et al. 2004, and this work). Our 2005 observations were made when E-P was at large heliocentric distances a few months after its aphelion. Only these and the 2000 observations made by Delahodde et al. (2004) report non-detected activity in the inbound part of the orbit. So, according to the recent observational evidence we predict that the comet-like activity of E-P is restricted to the post-perihelion orbit arc mentioned above.

\section{Rotational pole orientation}

Both the sense of rotation and the rotational pole orientation of E-P, which are closely related, are still unknown. We estimate the sense of rotation and the confinement of the direction of the rotational axis of E-P giving a limitation of the spatial 


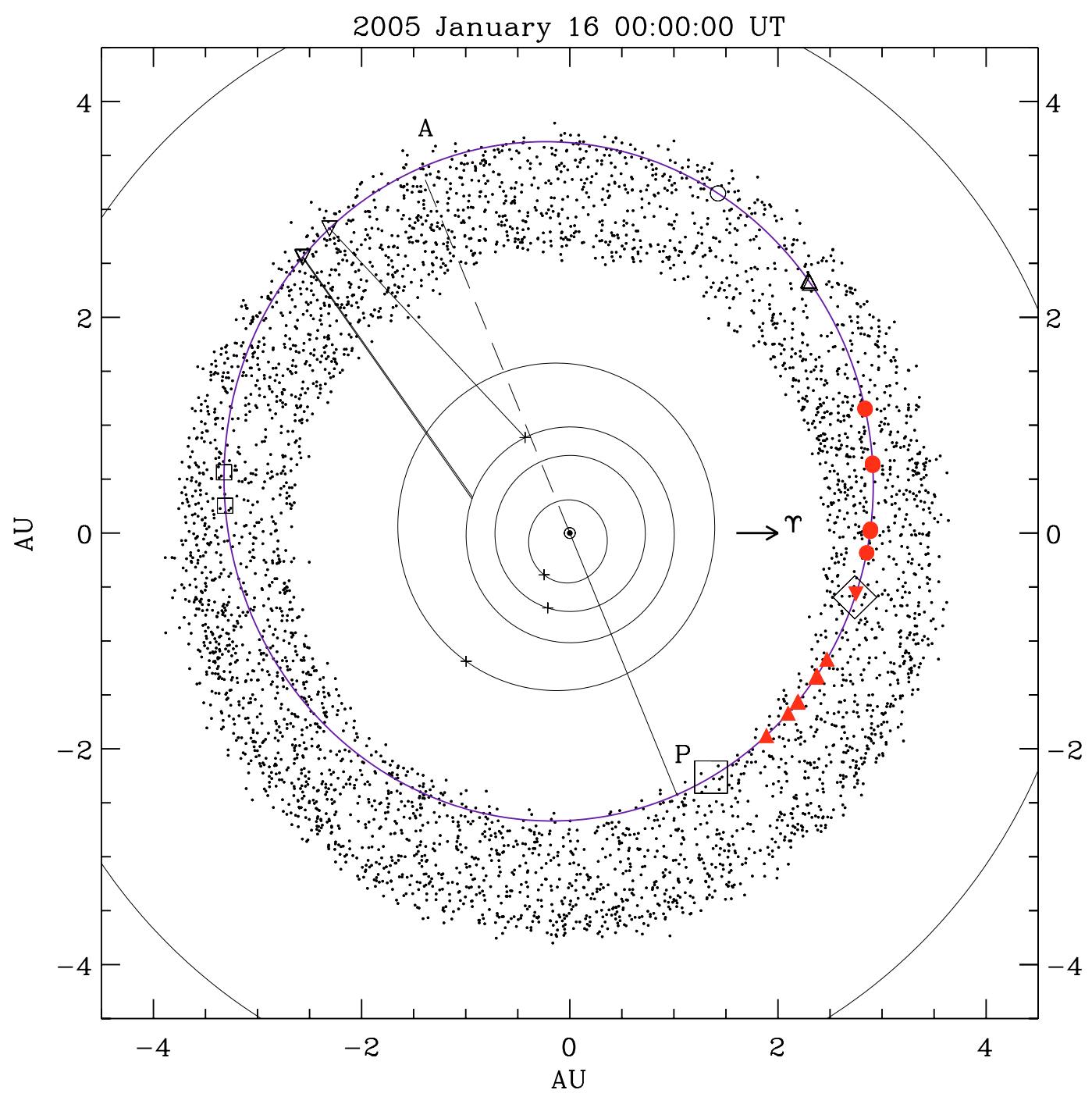

Fig. 3. Comparison of the active and dormant phases of the asteroid-comet transition object 7968 Elst-Pizarro (133P/Elst-Pizarro) in its orbit. The orbits and the instantaneous positions of the objects are projected onto the plane of ecliptic for the date of UT $0 \mathrm{~h} 16 \mathrm{January} 2005$. The orbit of 7968 Elst-Pizarro is drawn (solid blue line). Direction of its perihelion point $(\mathrm{P})$ is marked by a solid line connecting the Sun and the point in the orbit. Direction of aphelion (A) is marked by a dashed line. The solid line connects Earth and Elst-Pizarro on the date of the observations taken on UT 16 January, 28 February, and 1 March 2005, respectively. Background asteroids in the Themis-zone are also plotted (small black dots). The positions of the major planets from Mercury to Jupiter are marked with crosses. The orbits of major planets from Mercury to Jupiter are drawn with thin solid lines. The Vernal Equinox direction is indicated. Observations with reported dust trail (trail) activity are higlighted with solid red symbols: observations taken in 1996 are solid triangles, observations by Hsieh et al. (2004) from July to December 2002 are solid dots. Observation taken by Lowry \& Fitzsimmons (2005) in July 2002 is shown by a red triangle with downward directed peak, just near to the 1985 observation made by McNaught et al. (1996). Observations which did not report any comet-like activity are represented by open black symbols: large open square (1979), open diamond (1985), open triangles with upward peak (1997), small open squares (2000), open circle (2003), and open triangles with downward peak (this work). See also Table 2.

direction of the spin vector. Estimation of the sense of rotation is based on the examination of the temporal variation of the phase angle bisector (PAB) (Harris et al. 1984) during the lightcurve observations. As a byproduct of this method, the upper bound of the difference between the sidereal and synodic rotational periods is obtained. Guessing the possible direction vector of the rotational pole requires additional assumptions about the usual behavior of its temporal evolution, which is based on the conclusion of the results of the study of long-term evolution of the spin state and the rotational angular momentum vector direction of the nucleus of periodic comets. Samarasinha (1997) showed that the nucleus would reach an asymptotic spin state. In this case it is implicitly assumed that E-P behaves same way as a comet nucleus in terms of asymptotic behavior of its angular momentum direction. In addition, lightcurve and period analyses (Hsieh et al. 2004) showed that E-P rotates around its maximum moment of inertia i.e., its rotational state is not excited, consequently the angular momentum and spin axes coincide. We have many observations along the orbit of E-P which indicate whether this object was active or 
dormant. Comparing the locations of the active and dormant phases of E-P in its orbit (Fig. 3, Table 2), and taking into account the possible explanation of its seasonally recurrent activity that depends on the seasonally changing illumination and activation (Hsieh et al. 2004), we will estimate the limits of the rotational axis of E-P.

\subsection{Sense of rotation}

In order to determine or confine the sense of rotation we study the evolution of the phase angle bisector in the time for the full observational interval of E-P using the dates of observations of Hsieh et al. (2004). For example, if the PAB shows a monotonically increasing trend with time, in addition, assuming that the the synodic rotational periods are available for the sub-intervals of the full time span and these periods are monotonically increasing then the sense of rotation is prograde. It is described in more detail in Appendix of Harris et al. (1984) and it is based on the corrections in the basic formula of the photometric astrometry of asteroids (Taylor 1979; Taylor \& Tedesco 1983; and see examples for using of the PAB, which are given by Harris et al. 1984; Pravec et al. 1996; Toth 1997).

The direction of the motion of the PAB for E-P during the 2002 lightcurve observations (Hsieh et al. 2004) is displayed in the top panel of Fig. 4. There is a clear minimum in the time dependence of PAB around 1 October 2002, i.e., it is not a monotonic function. Its time derivative is shown in the mid panel of Fig. 4. If it was a monotonically increasing function then the sense of rotation could be prograde but it is not the case for those observations. However, it can help if full coverage nightly lightcurves are available and if investigation of the temporal changes in the synodic rotational period $\left(P_{\text {syn }}(t)\right)$ would show for example a monotonically increasing trend together with a monotonically increasing $\mathrm{PAB}$ in the time then the rotation is prograde. Unfortunately, it is not the case for the 2002 observations of E-P because there are no period determinations available for the nightly lightcurves but the period was derived by using all the observations (cf. Figs. 2 and 3 of Hsieh et al. 2004). There is a slight asymmetry in the motion of PAB (top panel of Fig. 4): for the observations made after 1 October 2002 the curve monotonically increases which supports the prograde rotation but this is only a slight indication of prograde rotation. However, there are no available constraints on the variation of $P_{\mathrm{syn}}(t)$ therefore we conclude that studying the temporal evolution of the PAB for the 2002 observations it is very difficult to definitely decide the sense of rotation of E-P and it still remains ambiguous.

In addition, the motion of the phase angle bisector causes uncertainty in the difference between the synodic $\left(P_{\text {syn }}\right)$ and the sidereal $\left(P_{\text {sid }}\right)$ periods of rotation (cf. Pravec et al. 1996). The maximum possible difference between the two types of periods $\left(\left|P_{\text {sid }}-P_{\text {syn }}\right| \leq|\Delta P|\right)$ is given by the formula

$\Delta P=\omega_{\mathrm{PAB}} P_{\mathrm{syn}}^{2}$

where $\omega_{\mathrm{PAB}}$ is the angular velocity of the PAB, i.e.,

$\left|P_{\text {sid }}-P_{\text {syn }}\right| \leq\left|\omega_{\text {PAB }}\right| P_{\text {syn }}^{2}$
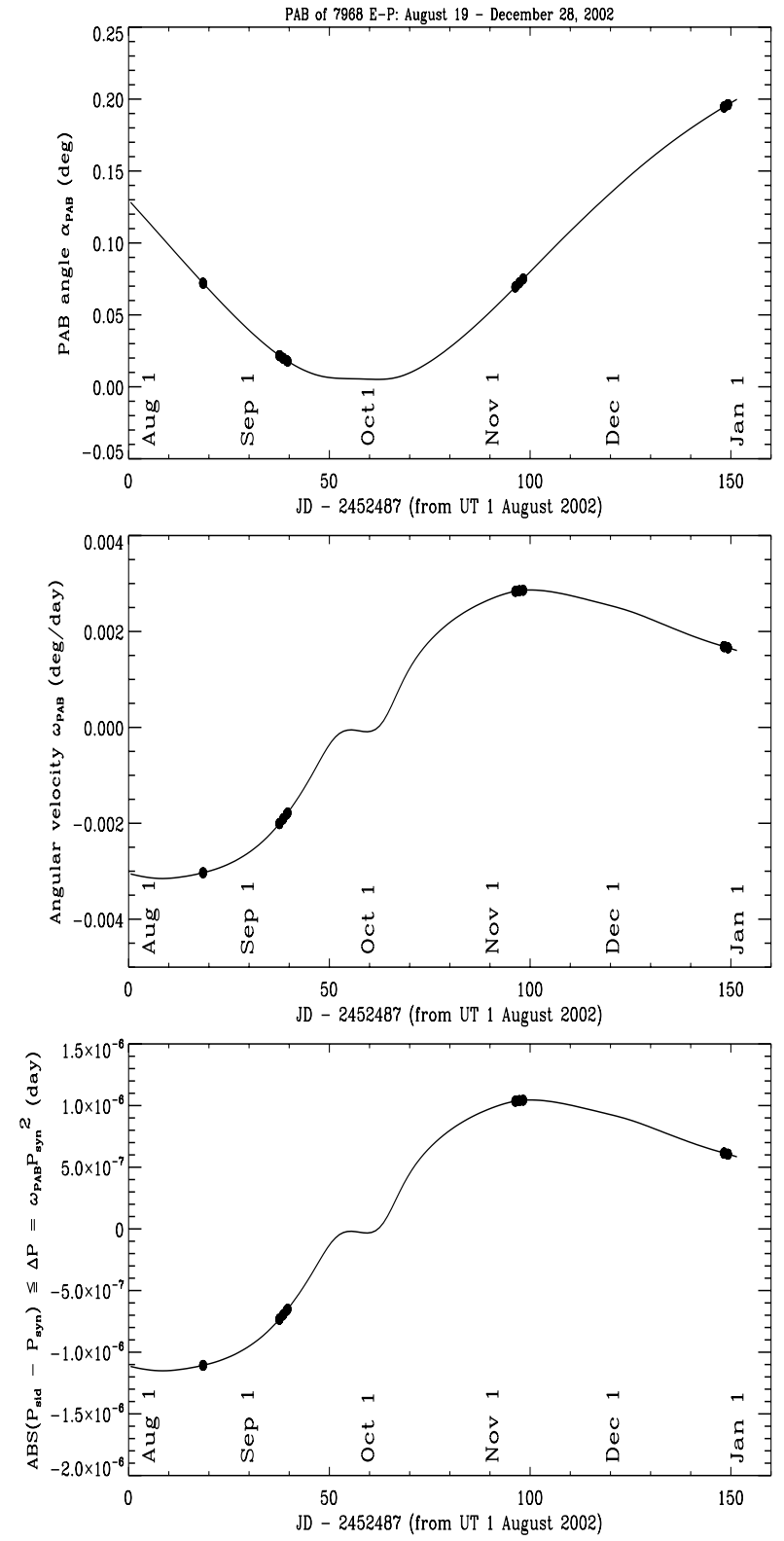

Fig. 4. Temporal evolution of the phase angle bisector (PAB) angle is displayed in the interval of the 2002 lightcurve observations by Hsieh et al. (2004) (top panel). Angular velocity $\omega_{\mathrm{PAB}}=\mathrm{d}(\mathrm{PAB}) / \mathrm{d} t$ of the phase angle bisector is shown in the same interval (mid panel). The angular velocity curve has ascending and descending branches but the monotonically ascending branch dominates the observation interval. The upper limit of the difference between the synodical and siderical rotational periods, which is limited by the angular velocity of the phase angle bisector and the square of the synodical rotational period (bottom panel). The difference is small: its order is $\sim 1.0 \times 10^{-6}$ day $(\sim 0.1 \mathrm{~s})$. Distribution of dates of the actual observations are indicated by dots in the curves.

where in the right hand side of the formula the absolute value of $\omega_{\mathrm{PAB}}$ is required because its sign can be changed during the PAB motion. In the case of the lightcurve observations made by Hsieh et al. (2004) the temporal change of $\Delta P$ has its maximum value at $\sim 1.0 \times 10^{-6}$ day $(0.000024$ h or $0.0864 \mathrm{~s})$ (bottom panel in Fig. 4). Consequently, the recently obtained rotational period values of $3.471 \pm 0.001 \mathrm{~h}$ (Hsieh et al. 2004) 
and $3.47113 \pm 0.00001 \mathrm{~h}$ (Delahodde et al. 2004) are close to the sidereal period of rotation but consequently both of these values can successfully be used in modeling.

\subsection{Orientation of the rotational axis - estimation and limitation of pole coordinates}

Estimation of the possible direction vector of the rotational axis of E-P requires additional assumptions about the usual behavior of its temporal evolution. Numerical integrations were carried out by Samarasinha \& Belton (1995) and Samarasinha (1997) for periodic comets over many orbits to study spin state evolution under outgassing induced torques. They have shown that the nucleus would reach an asymptotic spin state: there are preferred orientations for the rotational angular momentum vectors of periodic comets and the final rotational axis will be directed near one of these. Further investigations performed by Samarasinha (1997) again confirmed that the rotational angular momentum vector also asymptotically spirals towards a stable orientation in the inertial frame. An important consequence of this process is that the net torque on the nucleus will decrease. This spiralling toward the stable orientation is characterized by an accompanying decrease of the net torque on the nucleus in the inertial frame. It was concluded that two asymptotic stable orientations will evolve in the orbital plane: (i) near perihelion at the maximum cometary activity, and (ii) near aphelion at the orbital opposite of the maximum cometary activity. Consequently, we expect an excess of cometary rotational angular momentum vectors directed towards the perihelion or aphelion directions (Samarasinha 1997).

Regarding the rate of temporal change of spatial direction of the rotational angular momentum vector and the rotational state of the nucleus, Gutiérrez et al. (2002) studied the evolution of the rotational state of irregular shaped cometary nuclei. Their results show that the changes in the spin axis orientation can be large on long time scale but there are short time scale variations that are small at both the perihelion and large heliocentric distances. Accordingly, comparing the asymptotic behavior of the angular momentum vector orientation (Samarasinha 1997) and the temporal variations (Gutiérrez et al. 2002) the resulting asymptotic orientation has long-term stability. We assume that the rotational axis orientation of E-P has in a stable position for many orbits.

In order to confine the rotational pole direction in ecliptic coordinates we generated synthetic lightcurves assuming a rotating prolate ellipsoid shape model of E-P with semiaxes lengths of $a^{\prime}>b^{\prime}=c^{\prime}$ which rotates about its shortest axis (maximum moment of inertia) $c^{\prime}$. Each lightcurve was calculated at a given rotational pole direction which is parametrized by ecliptic longitude $L$ and latitude $B$ coordinates. Taking into account the aforementioned assumption of the asymptotic behavior of the rotational axis direction (Samarasinha \& Belton 1995; Samarasinha 1997) and the idea of the seasonal modulated activity model of E-P (Hsieh et al. 2004) the range of the ecliptic coordinates is naturally constrained by the distribution of the observed activity along the orbit, i.e., the post-perihelion orbit arc which is limited by the true anomaly range (see Fig. 3,
Table 2). The model parameters (dates of observations, semiaxes and rotational period of the body) are taken from Hsieh et al. (2004). The axial ratio $a^{\prime} / b^{\prime} \geq 1.45 \pm 0.07$ reported by Hsieh et al. (2004) is a lower limit only because the true aspect angle is unknown but we keep this value constant in our working model: it will not be a parameter to be fitted, i.e., we work with constant body sizes in searching for the pole direction. The quality of the fit at each pole coordinates $(L, B)$ was measured by the following $\chi^{2}$ parameter

$\chi^{2}(L, B)=\frac{1}{N} \sum_{i=1}^{N}\left[f_{\text {model }}\left(\phi_{i}\right)-f_{\text {obs }}\left(\phi_{i}\right)\right]^{2}$

where $f_{\text {model }}\left(\phi_{i}\right)$ is the model lightcurve point (magnitude), $f_{\text {obs }}\left(\phi_{i}\right)$ is the observation (magnitude). $N$ is the number of observations. The calculations are performed in the rotational folded phase plane at any rotational phase of $\phi_{i} \in[0,1]$. The resulting map of the $\chi^{2}(L, B)$ values in the plane of the ecliptic coordinates $(L, B)$ is displayed in Fig. 5. Of course we do not expect an unambiguous pole solution using a folded lightcurve constructed from a limited aspect geometry (cf. Fig. 3) but we obtained limits in the $(L, B)$ plane for the possible directions of the axis of rotation. For a comparison and guideline we also calculated a sine fit to the observed magnitude data and we found a maximum-minimum amplitude of $0.306 \pm 0.010 \mathrm{mag}$ (Fig. 6). It is the amplitude constraint for the aspect angle (pole orientation). We selected four best fit working models for the pole orientation of E-P, which are in good agreement with the observed data. These model lightcurves have maximumminimum amplitude values between 0.314 and 0.321 mag. The pole solutions of the heuristic models are summarized in Table 3. Both the calculated model curve and the curve of the sine fit are in good agreement for each model (see panels of Fig. 6). The pole solutions that are closer to the aphelion direction have $L=140^{\circ}$ and $B= \pm 60^{\circ}$ (Figs. 5 and 6, Table 3). These are proposed working models only and still we do not know the true solution for the rotational pole orientation of E-P unambiguously. Determination of the best pole solution can be made if more lightcurves are observed in many aspect geometric conditions. In addition, comparing the lightcurve observed by Hsieh et al. (2004) and a sine fit, as well as the rotating prolate ellipsoid model curves (Fig. 6), it can be recognized that the shape of E-P deviates from the prolate ellipsoid model, i.e., it is rather irregular, which is to be expected for small bodies.

\section{Conclusions}

1. Efforts were made to monitor Elst-Pizarro but our observations did not show any sign of activity on the nights of UT 16 January, 28 February and 1 March 2005.

2. To explain the recurrent activity of this asteroid-comet transition object we concluded that the locations of the active and dormant phases of Elst-Pizarro along its orbit support the idea of seasonally illuminated, activated and alternatively, dormant surface area.

3. Assuming that (i) E-P is a seasonally activated comet nucleus, and (ii) its angular momentum vector direction has an asymptotic behavior, we found that this vector is 

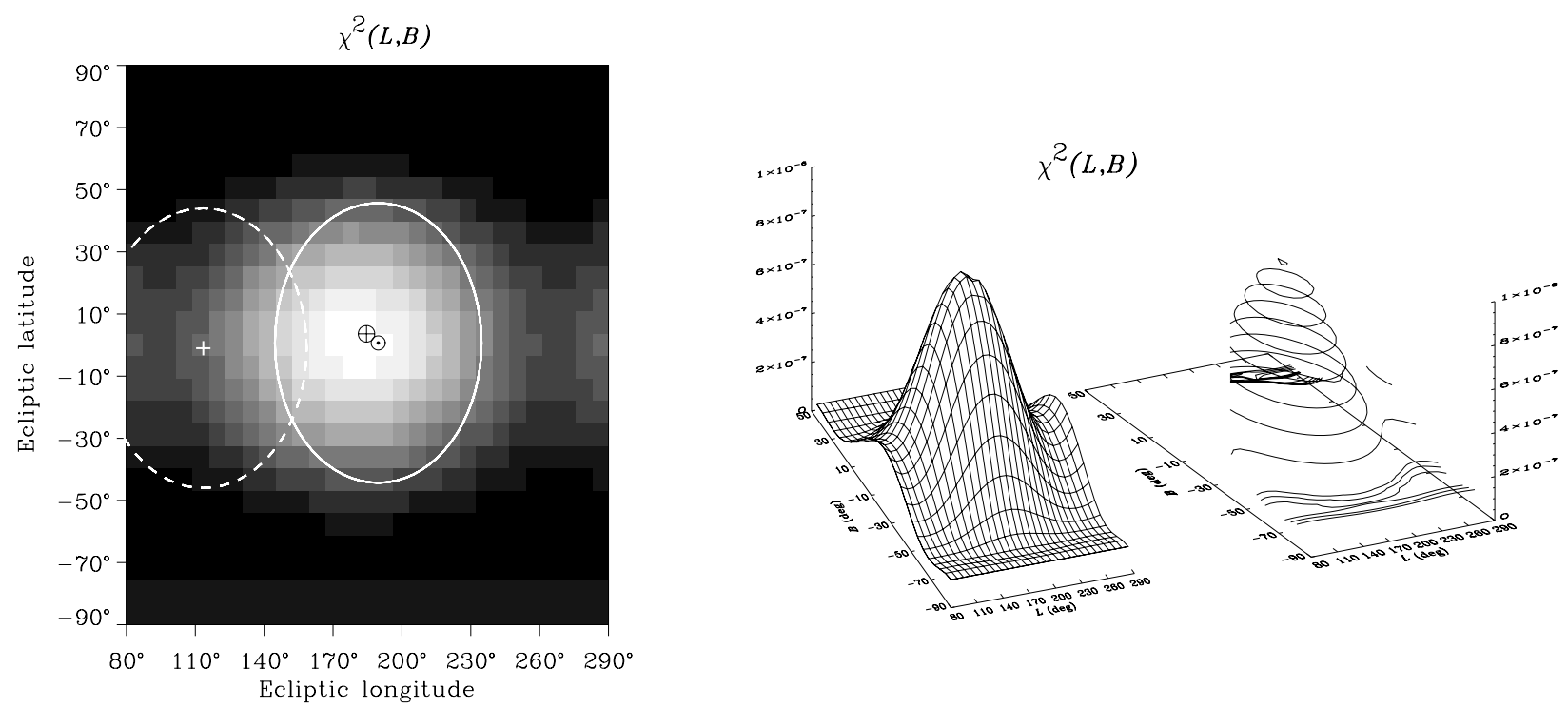

Fig. 5. Searching for possible pole directions in the ecliptic coordinate plane (longitude and latitude $(L, B)$ ) fitting the simulated lightcurves of the rotating ellipsoid model at different rotational pole orientations. Resulting $\chi^{2}$ values are displayed in the $(L, B)$ plane $(l e f t$ panel). The $\chi^{2}$ values are in the range of $4.8 \times 10^{-8}$ (darkest pixels) and $9.1 \times 10^{-9}$ (brightest pixels). Directions of Sun $(\odot$ ), Earth $(\oplus)$ viewing from E-P, and the direction of the aphelion (+) are marked. The limiting cone drawn around the solar direction is indicated by a closed solid curve and the limiting cone around the aphelion direction is plotted by a closed dashed curve. The $\chi^{2}$ values are also shown in 3-dimensional plots (right panel) both in shaded surface and wire-mesh representation. The best fit regions are at higher ecliptic latitudes both in the northern and southern hemispheres and limited by ecliptic longitudes of $80^{\circ}$ and $260^{\circ}$.

Table 3. Models of the rotational pole direction of 7968 Elst-Pizarro.

\begin{tabular}{ccccccc}
\hline \hline Model & $\begin{array}{r}L \\
\left({ }^{\circ}\right)\end{array}$ & $\begin{array}{r}B \\
\left({ }^{\circ}\right)\end{array}$ & $\begin{array}{r}\text { RA } \\
\left({ }^{\circ}\right)\end{array}$ & $\begin{array}{l}\text { Dec } \\
\left({ }^{\circ}\right)\end{array}$ & $\begin{array}{c}\xi \\
\left({ }^{\circ}\right)\end{array}$ & $\begin{array}{c}\text { Ampl. } \\
(\mathrm{mag})\end{array}$ \\
\hline (a) & 140 & +60 & 187 & +67 & 66 & 0.315 \\
$(\mathrm{~b})$ & 220 & +60 & 239 & +42 & 66 & 0.314 \\
$(\mathrm{c})$ & 140 & -60 & 121 & -42 & 68 & 0.321 \\
$(\mathrm{~d})$ & 220 & -60 & 173 & -67 & 68 & 0.320 \\
\hline
\end{tabular}

Note:

$L, B$ : ecliptic latitude and longitude (2000).

RA, Dec: equatorial coordinates (2000).

$\xi$ : aspect angle of the pole.

Ampl: maximum-minimum amplitude.

See also Fig. 6.

probably directed towards the aphelion of E-P's orbit. In addition, taking into account the amplitude of the observed lightcurve in 2002, as well as the derived prolate ellipsoid body shape using the data given by Hsieh et al. (2004), we estimated the possible ecliptic coordinate limits of the vector of the rotational axis. We constructed working models for the possible orientation of the rotational pole, which are still ambiguous heuristic models. The calculated model lightcurves fit the observations well. Those pole solutions are closer to the aphelion direction which have ecliptic coordinates $L=140^{\circ}, B=+60^{\circ}$ or $-60^{\circ}$.

4. The sense of rotation still cannot be determined definitely, however, there is a slight indication that it is prograde. Moreover, from the range of motion of the phase angle bisector we estimated that the the derived rotational period and the sidereal period of rotation of E-P are very close: these are within $\sim 10^{-5} \mathrm{~h}$,

5. Our recent analysis of the lightcurve observed by Hsieh et al. (2004) shows that the shape of E-P deviates from the prolate ellipsoid model and it is rather irregular.

6. There are certain caveats about the recent conclusion about the seasonal activity of E-P: (i) the observations made in the inbound and outbound parts of the orbit are not equal: there are only two sets of pre-perihelion observations (inbound arc of the orbit) Delahodde et al. (2002) and this work. We presume that the activity is limited to the post-perihelion arc of the first angular quarter in true anomaly. It should be confirmed by more observations that in the inbound part of the orbit the object remains dormant; (ii) the outgassing activity has not been directly observed yet, only the released dust trails (or tail) have been observed. But a true comet has to be gas activity, e.g., gas coma, spectral features of gases, the presence of a persistent gas coma which fits the comet definition; (iii) there are not enough lightcurve observations to constrain the shape of E-P and the rotational pole orientation and sense of rotation.

7. In order to improve the knowledge about this activated asteroid there will be new opportunities during its 2006-2007 visibility periods. The next perihelion of Elst-Pizarro will be on 29 June 2007 when the object will be at a perihelion distance of $q=2.634$ AU. According to the 1996 and 2002 activity period (Fig. 3) we predict an analogous time schedule for restarting of its active period. At that time the visibility of E-P will be favorable for ground-based observers in the southern hemisphere but the object will be close to the galactic equator where the crowding by star-fields is unfavorable. 

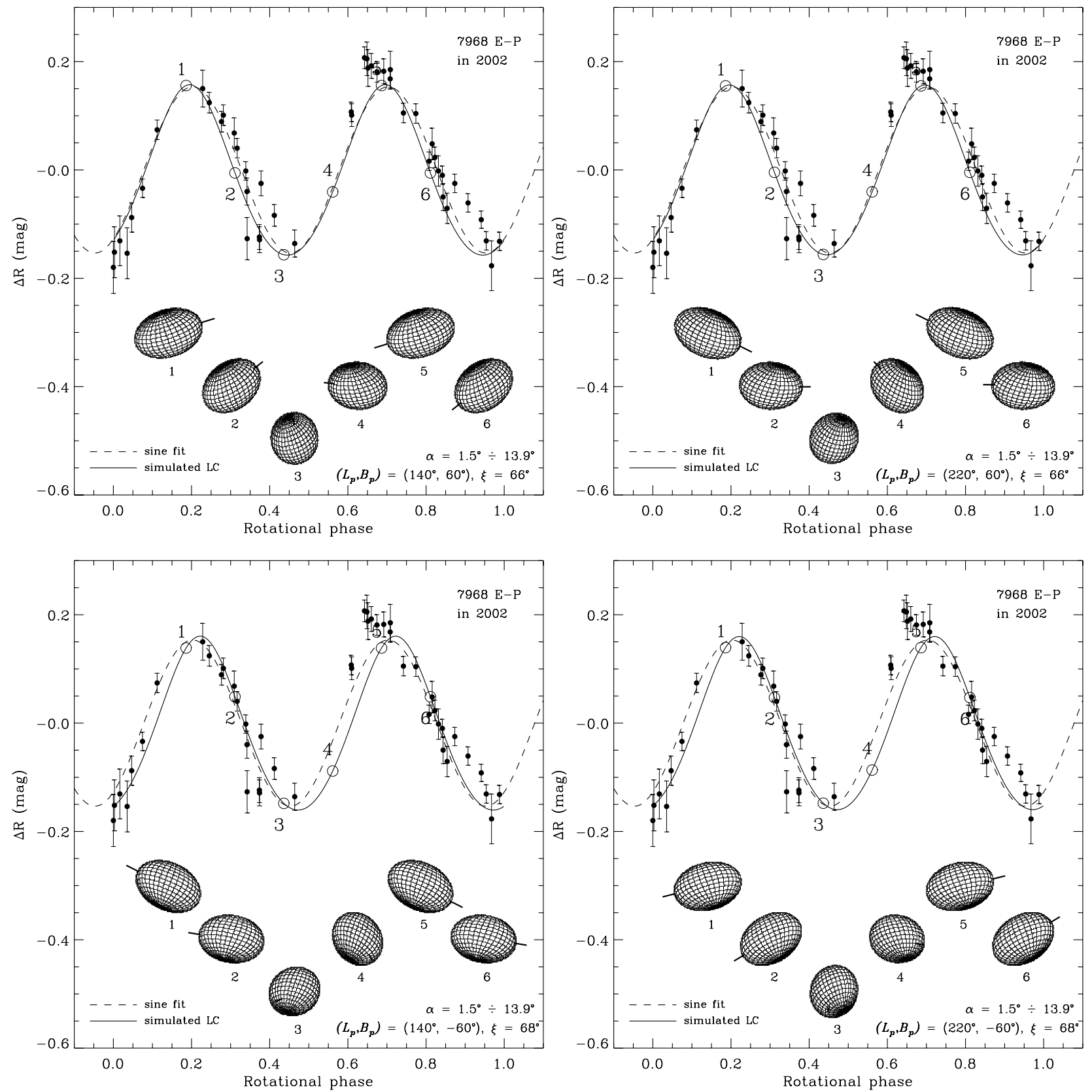

Fig. 6. Comparison of the lightcurves of E-P. The data points (dots) observed in 2002 (Hsieh et al. 2002) are reduced $R(1,1,0)$ magnitudes shifted to zero mean are shown $(\Delta R)$. A few outliers were omitted. The sine fit to the data with minimum to maximum amplitude of $0.306 \pm$ 0.010 mag is plotted (dashed line). The parameters of the model body shape are given taken from Hsieh et al. (2004). The rotational pole is selected taking into account the $\chi^{2}$ domain constrains for the best fit lightcurves (cf. Fig. 5). The ecliptic coordinates $\left(L_{p}, B_{p}\right)$ of the rotational pole, aspect angle $\xi$, and the solar phase angle $\alpha$ range of the observational span are shown. The rotating model body is drawn at different rotatinal phases as seen from Earth (numbers identify the position of the corresponding points in the lightcurve). Since the solar phase angle is small therefore about the same surface part is illuminated by the Sun wich can also be seen from Earth. Four panels correspond to Table 3: top panels are the models a) and b), bottom panels are the models $\mathbf{c}$ ) and $\mathbf{d}$ ), respectively. The rotational pole has northern ecliptic latidude in the top panels, and it has southern ecliptic latitude in the bottom panels. The maximum-minimum amplitudes of the calculted model lightcurves are as follows: $0.315 \mathrm{mag}$ (top left), $0.314 \mathrm{mag}$ (top right), $0.321 \mathrm{mag}$ (bottom left), 0.320 mag (bottom right).

Acknowledgements. The author thanks Henry Hsieh and David C. Jewitt of Institute for Astronomy of University of Hawaii for the beneficial discussions on asteroid-comet Elst-Pizarro, in addition, Zoltán Csubry of Konkoly Observatory for the advices, in using of the Roper CCD camera control software. This work used the ASTORB data base which is maintained by Edward L. G. Bowell of the Lowell Observatory.

\section{References}

Boehnhardt, H. 1996, IAU Circ., 6495

Boehnhardt, H. 1997, MPE Circ., 1997-T03

Boehnhardt, H., Sekanina, Z., Fiedler, A., et al. 1998, Highlights Astron., 11A, 233

Delahodde, C. E., Hainaut, O. R., Dotto, E., \& Campins, H. 2004, BAAS, 36, 1148 
Elst, E. W., Pizarro, O., Pollas, C., et al. 1996, IAU Circ., 6456

Fernández, Y. R., McFadden, L. A., Lisse, C. M., Helin, E. F., \& Chamberlin, A. B. 1997, Icarus, 128, 114

Gutiérrez, P. J., Ortiz, J. L., Rodrigo, R., López-Moreno, \& Jorda, L. 2002, EMPl, 90, 239

Hammergren, M. 1996, BAAS, 28, 1299

Harris, A. W., Young, J. W., Scaltriti, F., \& Zappalá, V. 1984, Icarus, 57,251

Hsieh, H. H., Jewitt, D. C., \& Fernández, Y. R. 2003, BAAS, 35, 1011 Hsieh, H. H., Jewitt, D. C., \& Fernández, Y. R. 2004, AJ, 127, 2997

Jewitt, D. 2004, in Comets II, ed. M. C. Festou, H. U. Keller, \& H. A. Weaver (Tucson: University of Arizona Press), 659

Jewitt, D. C. 2005, AJ, 129, 530

Lien, D. J. 1998, BAAS, 30, 1035

Lowry, S. C., \& Fitzsimmons, A. 2005, MNRAS, 358, 641

Marsden, B. G. 1996, IAU Circ., 6457

Marsden, B. G., \& McNaught, R. H. 1996, MPE Circ., 1996-R07
McNaught, R. H., Hawkins, M. R. S., Marsden, B. G., et al. 1996, IAU Circ., 6473

Offutt, W. 1996, IAU Circ., 6456

Offutt, W. 1997, MPE Circ., 1997-T03

Pravec, P. 1996, IAU Circ., 6459

Pravec, P., Šarounová, \& Wolf, M. 1996, Icarus, 124, 471

Samarasinha, N. H., \& Belton, M. J. S. 1995, Icarus, 116, 340

Samarasinha, N. H. 1997, BAAS, 29, 743

Taylor, R. C. 1979, In Asteroids, ed. T. Gehrels (Tucson: University of Arizona Press), 480

Taylor, R. C., \& Tedesco, E. F. 1983, Icarus, 54, 13

Tichá, J., \& Tichý, M. 1996, IAU Circ., 6456

Toth, I. 1997, Planet. Space Sci., 45, 1625

Toth, I. 2000, A\&A, 360, 375

Weissman, P. R., Bottke, W. F. Jr., \& Levison, H. F. 2002, in Asteroids III, ed. W. F. Bottke Jr., A. Cellino, P. Paolicchi, \& R. P. Binzel (Tucson: Univ. of Arizona Press), 669 